\title{
Topological Space of the Knee Tensegrity System
}

\author{
Wangdo Kim* and Andrew Park \\ School of Mechanical and Aerospace Engineering, Seoul National University, Seoul Korea
}

Received: March 09, 2018; Published: March 19, 2018

*Corresponding author: Wangdo Kim, School of Mechanical and Aerospace Engineering, Seoul National University, Seoul Korea, Tel: +82-10-8644-2145; Email: mwdkim@gmail.com

\begin{abstract}
Estimating the knee axis is crucial to both correctly aligning components in knee arthroplasty and assessing the joint kinematics. From the point of view of perceptual psychology, it is especially interesting that one can use the part-whole relationship at the knee joint as the basic theory. We characterize the concept of a "knee axis" and further the concept of "surrounding." It is now generally recognized that the whole-part relationship, and the relationships of the parts to each other play a fundamental role in producing the screw axis of the knee (SAK). We present conditions of component alignment in the arthroplasty based on the principle that the part should become a member of the whole. If any screw motion with respect to the SAK is applied to the lines of forces that form the surrounding, these forces remain within the surroundung.
\end{abstract}

Keywords: knee arthroplasty; screw axis of the knee; knee tensegrity; knee topology

Abbreviations: TKA: Total Knee Arthroplasty; KSA: Knee Screw Axis; KFA: Knee Functional Axes; ACL: Anterior Cruciate Ligament; PCL: Posterior Cruciate Ligament; MCL: Medial Collateral Ligament ; JRS: Joint Reaction Subspace

\section{Introduction}

A good outcome in total knee arthroplasty (TKA) depends on many factors, including joint alignment, range of motion, patellar tracking, and ligament stability. It is now generally recognized that knee axes are used for both component alignment in knee arthroplasty Feng, Tsai et al. [1] and ideal placement of the prosthesis during knee reconstructive surgery Kim, Chung et al. [2]. Consequently, estimating of the knee axis is one of the key topics for the "2010 ASME Grand Challenge Competition to Predict in Vivo Knee Loads" Fregly, Besier et al. [3]. Knee Functional Axes (KFA) information is referred to the knee screw axis (KSA) Kim, Kim et al. [4-6]. In that case, the intersegmental force such as ligaments and contact forces are in pure tension/compression and are surrounding the KSA in such a way that those forces result in no (virtual) works Kim, Veloso et al. [7]. This basic theorem from the topological space states that any two objects, U and V, of a system for which certain conditions hold, the following relationship shall or shall not be valid: $U$ is a part of $\mathrm{V}$ (that is equivalent to "V includes U") Lewin Mammen et al. [8] Therefore the aims of this paper are to characterize the concept of a "knee axis" (U) and further the concept of "surrounding (V)."

\section{Materials and Methods}

The intra-articular structures of the tensegrity system of the knee include the muscles, the anterior cruciate ligament (ACL), posterior cruciate ligament (PCL), medial collateral ligament (MCL), and articular contact in the medial (P1) and lateral (P2) compartments (Figure 1) Kim Veloso et al. [9]. We have shown that six constraints are members of the "joint reaction subspace (JRS)," and are spatially oriented in such ways that by imposing an internal tension or "prestress" to reduce the freedom in the system; this ensures immediate mechanical responsiveness (i.e., that movement of one element is felt by all others) and reduces impact fatigue at the joint Kim Veloso et al. [10]. Architects call this type of prestressed structural network, composed of opposing tension and compression elements that self-stabilizes its shape through establishment of a mechanical force balance, a tensegrity (tensional integrity) structure (Figure 1). Alternatively, this means that JRS does not generate power Kim Veloso et al. [10-12]. Biotensegrity is a term introduced by Dr. Stephen Levin, and denotes the application of tensegrity's principles to biological structures Hutson Ellis et al. [13]. 


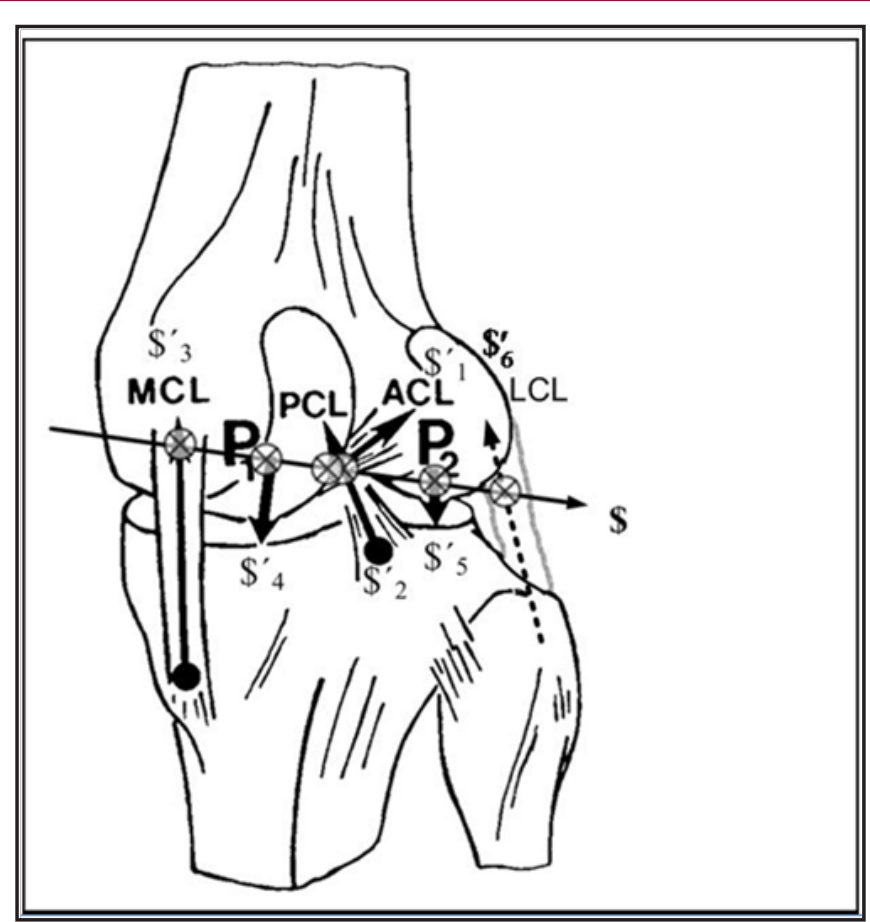

Figure 1: The tensegrity structure of surrounding forces applied to the knee joint: native system of the knee where six constraints are collectively reciprocal to the KSA indicated by $\$$. That the virtual coefficient should vanish is necessary, and the sufficient conditions Kim, Veloso et al. [9], or the pair (and) are in involution Kim, Chung et al. [6]. The tensegrity's structure is characterized by the contact normal elements $\$_{4}^{\prime}$ and $\$^{\prime}{ }_{5}$, while all the other elements are, continuous tension elements.

\section{Results and Discussion}

The present research addressed a multifractal tensegrity structure Turvey Fonseca et al. [14] by adopting the topological space at the knee. Anatomically derived axes have been used to investigate the knee kinematic Feng, Tsai et al. [1]. In this paper, however, we have been concerned with those properties which remain unchanged, or "invariant", under a special class of transformation which lies under the tensegrity system of the knee. Hence if the surrounding structure of the whole is subjected to a screw motion about the SAK, the whole itself is not altered, and we can say this is the unit of movement during locomotion. This is the class of "topological space of the knee". Although the simplicity of the knee axis has the significant advantage for robotic system, this approach has significant disadvantage for biologically inspired system (e.g., prosthetics). The current review has proposed if tensegrity based reconstruction surgery could be designed for the next generation arthroplasty and prosthetic device.

\section{References}

1. Feng Y, TY Tsai, JS Li, HE Rubash, G Li, et al. (2016) In-vivo analysis of flexion axes of the knee: Femoral condylar motion during dynamic knee flexion. Clin Biomech (Bristol, Avon) 32: 102-107.

2. Kim W, H Chung (2016) Proposed Tibial Femoral Tunnel for Isokinetic Graft Placement: A Mathematical Model. Annals of Sports Medicine and Research 3(8): 6.

3. Fregly BJ, TF Besier, DG Lloyd, SL Delp, SA Banks, et al. (2012) Grand challenge competition to predict in vivo knee loads. Journal of Orthopaedic Research 30(4): 503-513.
4. Kim W, AP Veloso, D Araújo, V Vleck, F João (2013) an informational framework to predict reaction of constraints using a reciprocally connected knee model. Computer Methods in Biomechanics and Biomedical Engineering p: 1-12.

5. Kim W, Y Choi, HG Lee (2016) the Duality of Knee Functional Axes and Foot Contact. Journal of Functional Morphology and Kinesiology 1(4): 387-395.

6. Kim W, Y Choi, H Lee (2016) Observations on the Knee Functional Axis During Active Movements. SM Musculoskeletal Disorders 1(1): 5.

7. Kim W, AP Veloso, VE Vleck, C Andrade, SS Kohles (2013) The stationary configuration of the knee. J Am Podiatr Med Assoc 103(2): 126-135.

8. Lewin K (1969) Principles of topological psychology. Johnson Reprint Corp, New York, USA.

9. Kim W, A Veloso, J Tan, C Andrade (2010) A Reciprocal Connection at Knee Joint. ASME 2010 Summer Bioengineering Conference, USA, pp: 263-264.

10. Kim W, YH Kim, AP Veloso, SS Kohles (2013) Tracking Knee Joint Functional Axes through Tikhonov Filtering and Plűcker Coordinates. Journal of Novel Physiotherapies 4(1).

11. Kim W, M Espanha, A Veloso, D Araújo, F João (2013) An Informational Algorithm as the Basis for Perception-Action Control of the Instantaneous Axes of the Knee. J Nov Physiother 3(127): 2.

12. Hutson MA, RM Ellis (2006) Textbook of musculoskeletal medicine. Oxford, Oxford University Press, $2^{\text {nd }}$ Edition New York, USA.

13. Turvey MT, ST Fonseca (2014) The medium of haptic perception: a tensegrity hypothesis. J Mot Behav 46(3): 143-187.

14. Mammen J (2016) Using a Topological Model in Psychology: Developing Sense and Choice Categories. Integr Psychol Behav Sci 50(2): 196-233. 


\section{(C) (i) This work is licensed under Creative}

Submission Link: https://biomedres.us/submit-manuscript.php

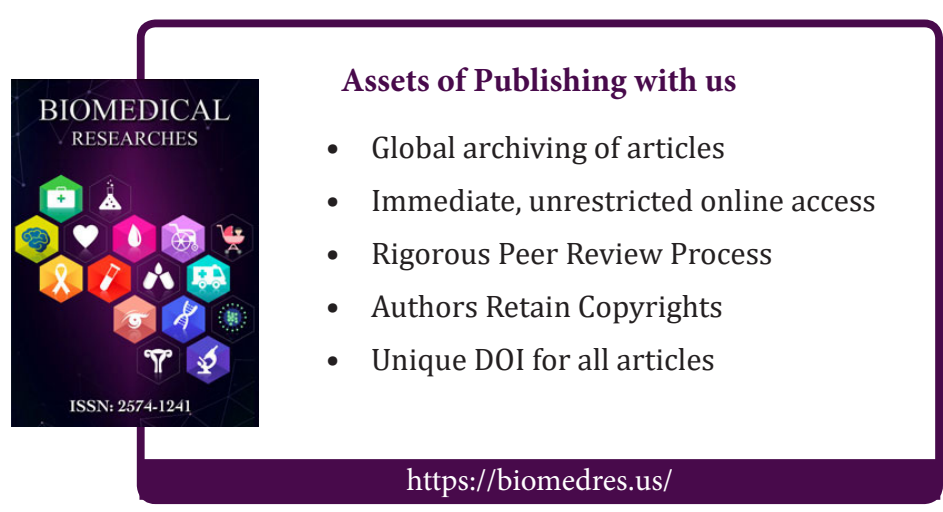

